\title{
Applying a Common-Sense Approach to Fighting Obesity
}

\author{
Jessica Y. Breland, ${ }^{1,2}$ Ashley M. Fox, ${ }^{3}$ Carol R. Horowitz, ${ }^{3}$ and Howard Leventhal ${ }^{1,2}$ \\ ${ }^{1}$ Institute for Health, Healthcare Policy and Aging Research, Rutgers, The State University of New Jersey, New Brunswick, \\ NJ 08901, USA \\ ${ }^{2}$ Department of Psychology, Rutgers, The State University of New Jersey, New Brunswick, NJ 08901, USA \\ ${ }^{3}$ Mount Sinai School of Medicine, One Gustave L. Levy Place, P.O. Box 1077, New York, NY 10029, USA
}

Correspondence should be addressed to Jessica Y. Breland, jessica.breland@gmail.com

Received 16 December 2011; Revised 25 April 2012; Accepted 9 May 2012

Academic Editor: Jutta Mata

Copyright (c) 2012 Jessica Y. Breland et al. This is an open access article distributed under the Creative Commons Attribution License, which permits unrestricted use, distribution, and reproduction in any medium, provided the original work is properly cited.

\begin{abstract}
The obesity epidemic is a threat to the health of millions and to the economic viability of healthcare systems, governments, businesses, and nations. A range of answers come to mind if and when we ask, "What can we, health professionals (physicians, nurses, nutritionists, behavioral psychologists), do about this epidemic?" In this paper, we describe the Common-Sense Model of Self-Regulation as a framework for organizing existent tools and creating new tools to improve control of the obesity epidemic. Further, we explain how the Common-Sense Model can augment existing behavior-change models, with particular attention to the strength of the Common-Sense Model in addressing assessment and weight maintenance beyond initial weight loss.
\end{abstract}

\section{The Magnitude and Distribution of the Problem}

Almost two-thirds of adults in the United States are overweight (body mass index; BMI $\geq 25 \mathrm{~kg} / \mathrm{m}^{2}$ ) or obese (BMI $\geq$ $30 \mathrm{~kg} / \mathrm{m}^{2}$ ) [1] , and rates of overweight and obesity have increased considerably since the 1980s [1]. Although the trend appears to be slowing [1], it remains disturbing as obesity is associated with numerous chronic illnesses, including type 2 diabetes, hypertension, respiratory problems, and various cancers. It is also the second leading cause of preventable death in the United States [2]. Worldwide, roughly 1 billion people were overweight or obese in 2010 and that number is expected to grow to 1.5 billion by 2015 [3]. Further, overweight and obesity are linked to a large portion of the chronic disease burden in many countries and now account for more deaths than underweight [3]. Overweight and obesity are also associated with significant psychological burden as these patients often face and internalize considerable stigma [4]. Additionally, treating obese patients costs roughly $\$ 1,429$ more than treating normal weight patients and the cost of obesity to the US medical system approached $\$ 150$ billion in 2008 [5].

\section{Treating Obesity}

Given the social, health, and economic burden of obesity and overweight, numerous interventions have been designed for obese patients. These interventions usually recommend a combination of diet and/or physical activity with behavioral support, but can also include surgery and/or medication [6]. Although many of these interventions result in weight loss (e.g., [6-11]), available data raise two critical questions: (1) is the weight loss clinically significant? And (2) can it be maintained? A recent thorough review of weight loss interventions for overweight or obese adults [6] suggests that the answer to the first question is unclear as there is no consensus on the definition of clinically significant weight loss. With respect to the second question, the review suggests that many patients are unable to maintain weight loss for more than 3 years [6]. Additionally, successful programs, such as the Diabetes Prevention Program [12], are too costly and time intensive to be implemented outside of academic settings [13]. Further, interventions conducted in standard care settings have had poor results; for example, attempts to train primary care physicians to provide brief weight loss counseling have been relatively unsuccessful [14]. 


\section{Conceptual Framework for the Control of Obesity: A Common-Sense Approach}

Although a "one size fits all" approach makes little sense for controlling the obesity epidemic, it would be useful to identify a set of underlying self-regulation principles common to both population and individual, behavioral approaches for prevention and intervention. If such principles exist, it would create an opportunity to integrate "public" messages for prevention and the messages and interventions delivered by individual practitioners, and to increase the impact of both. The Common-Sense Model of Self-Regulation (CSM) [15], a model of the cognitive and affective mechanisms underlying chronic illness management, provides a set of concepts and tools we believe will be useful for this integration. The CSM translates the intra- and interpersonal processes of illness management into objective indicators and as such is a useful tool for generating and testing hypotheses. The diabetes world has found the CSM useful for promoting blood glucose monitoring and management $[16,17]$, and it may prove to be as useful for the treatment of obesity. However, in order for any theory to effectively inform research and practice, it must be understood and used properly. Therefore, the goal of this commentary is to present the CSM in such a way that it will inspire and be used in future work related to weight management. First, we describe the CSM and how it could be used to augment the assessment and long-term management strategies used in current behavioral treatments. Next, we discuss studies that have investigated the CSM and obesity. Lastly, we describe how the CSM might be used in clinical practice and research.

\section{The CSM and Assessment}

At its core, the CSM suggests that patients use information from the following five domains to understand and respond to health threats (e.g., obesity): (1) the identity of the threat (e.g., labeling excess body fat "obesity"); (2) the cause of the threat (e.g., overeating or inactivity); (3) the consequences of the threat (e.g., heart disease or stigma); (4) whether the threat can be controlled/cured (e.g., believing dieting is effective); (5) the time line associated with the threat (e.g., whether obesity is viewed as an acute or chronic condition or whether it develops and changes rapidly or slowly). Patients' ongoing experiences in these domains develop illness representations, personal "common-sense" understandings of conditions. Numerous findings have demonstrated that illness representations are associated with and predictive of a variety of health behaviors [18]. A more recent meta-analysis of research with cardiac patients, whose treatment often includes changes in diet and exercise, demonstrated that patients with strong beliefs in the control, identity and consequence domains were more likely to attend cardiac rehabilitation [19]. Further, Petrie et al. [20] found that an intervention addressing cardiac patients' illness representations reduced pain and time spent away from work.

Patients use similar domains to develop representations of treatment based on experiences with self-generated and prescribed treatments. These experiences and the resulting representations are modified by the illness's representation and threat level. For example, asthma patients who believe they only have asthma when they have symptoms are less likely to use maintenance inhalers [21] — why take a chronic medication for an "acute" problem? In fact, it has been suggested that the link between illness representations and asthma is strong enough to merit a Cochrane Review [22]. The CSM suggests that the results would be similar for obesity [23]; for example, obesity interpreted as a result of poor eating habits might result in a change in diet; whereas obesity interpreted as the result of stress may be seen as inevitable and untreatable. That is, the links between illness and treatment representations are not random; they are based on a "common-sense" match between the perceived cause of the illness and the treatment perceived as most likely to address that underlying cause. "Common-sense matches" activate expectations in each of the five domains; that is, the symptoms and/or functions that will improve, the rapidity of change, the need to continue action to maintain achieved benefits, and expectations about experienced consequences, benefits, and losses, over time from both treatment and illness.

Thus, the CSM suggests that in order for weight loss interventions to be effective, they must either match patients' illness and treatment representations of obesity or provide a compelling case for patients to change their illness and/or treatment beliefs. In this way, the CSM could be used to augment existing obesity treatments during the behavioral assessments that have been a core component of behavioral treatments for decades [24]. Careful assessment of the presenting problem facilitates the identification of appropriate treatment targets and assessing patients early and often allows clinicians to track early responses to treatment, a factor highly correlated with positive outcomes [25]. The CSM's five illness and treatment domains are associated with health behaviors and could therefore provide clinicians with a roadmap for this assessment. The domains map onto areas routinely assessed by physicians during medical visits, making them easy to add to usual care. Initial studies report that primary care physicians favorably view addressing patient goals and using action plans, which are key components of the CSM [26]. Further, patients are more satisfied when their physicians address illness and treatment representations [27] and a recent study reported better adherence to treatment one-month after-visit for patients whose internists addressed illness and treatment representations [28]. Some of the latter study's participants were prescribed diet and physical activity changes, suggesting that assessing and addressing illness and treatment beliefs could improve adherence to weight loss treatments. Additional studies are clearly needed.

\section{The CSM and Weight Maintenance}

According to the CSM, illness and treatment representations function in relation to interactions among the various feedback systems responsible for human behavior. Some of these feedback systems involve partially or fully conscious, deliberative decisions (e.g., reading and interpreting food 
labels). These conscious and deliberative systems are the focus of our conversations about everyday actions, such as discussions about diet between a patient and a practitioner and messages about diet on television or menus. These deliberative feedback systems are also of central concern to investigators examining the effects of medical/health literacy on the understanding of treatment, decision making, and adherence to lifestyle changes.

However, while these conscious deliberative processes are the focus of many obesity interventions [6], the CSM suggests that conscious/deliberative activity regulates but a small fraction of the everyday behaviors associated with weight loss. Rather, much of human behavior is driven by systems that function automatically (e.g., one does not consciously decide to digest food) or, more importantly, are habitual (e.g., the semiautomatic sequences involved in bringing a fork to one's mouth). For example, making the decision to go out to a fast food venue for dinner involves at least one conscious decision; however, the actions involved in going out to dinner are mostly automatic and semiautomatic habitual behaviors (e.g., the sensation of hunger, traveling to the restaurant, putting food into one's mouth, finishing a large meal without attending to cues of satiety). In short, most of what happens in everyday life involves welllearned habits that can initiate and maintain behavior with little deliberative decision making. Thus, examining obesity through the lens of CSM control systems, suggests that altering food intake and physical activity to prevent and treat obesity requires replacing existent, potentially "toxic" automatic procedures with procedures associated with healthy outcomes.

In other words, the CSM suggests that overweight and obese patients must replace habitual patterns in everyday life that lead to weight gain with those that do not. Planning (i.e., observing and/or mapping) daily life sequences for eating-beginning with where one shops and what one buys, through how food is prepared, plated and eaten and replacing harmful with helpful actions-is at the "commonsense" core of creating action plans for weight loss. The CSM suggests that the detection of changes both at a given time and most importantly over time, with clear records based on function, somatic sensations, and/or pictures, are critical for motivating behavioral change at the outset and most importantly, for sustaining it over time. The significance of daily and ongoing benchmarks is detected in everyday comments such as, "It was a bad day," and "I barely had enough energy to make it through," "I skipped breakfast and had a smaller than usual lunch and I'm still hungry." Providing benchmarks for these momentary appraisals of the daily self and a clear vision of changes over time can affect behaviors, such as uncontrolled late night snacking, that are critical for weight regulation. The focus on addressing habitual and deliberate behavior is similar to cognitive behavioral therapy-(CBT-) based weight loss treatments that attempt to increase awareness of habitual behaviors through self-monitoring [6] informing the compatibility of the CSM with traditional CBT.

Also similar to behavioral approaches (e.g., [29]), the CSM suggests that replacing unhealthy behaviors with healthy behaviors requires examining an individual's daily environment to identify: (1) the initiating and maintaining factors for unhealthy and healthy eating and sedentary and active behaviors and (2) available sources of non-toxic foods and safe places to engage in physical activity every day. However, some of the earliest findings regarding the CSM [30] demonstrated that action plans based on these steps are not sufficient for behavior change. Rather, action plans need to be linked to motivating representations, for example, evidence that the self is less symptomatic (e.g., better joint function) or exposed to lower disease risk (e.g., improved blood pressure). The need to combine action plans with concrete evidence that motivates has been replicated numerous times [31] and suggests a third step necessary for weight loss and maintenance: to identify outcomes associated with toxic foods and sedentary activities, increase their negative valence and identify and increase the positive valence of outcomes associated with healthy foods and physical activity. Physician visits present an opportunity to address these three steps as well as an opportunity to devise a tailored action plan for change, as described in the next section.

As with other effective weight loss theories [6], the CSM suggests that weight loss must be presented and achieved in a step-by-step fashion, that is, patients must recognize that change takes time. However, one major difference between the CSM and other theories is the role of constant feedback in developing long-term action plans that will maintain behavioral changes. A potentially novel contribution of the CSM is the hypothesis that a sole focus on weight loss will make weight maintenance problematic because weight changes too slowly and fluctuates too often to serve as an effective daily reinforcer. The CSM suggests that long-term change requires more than simply telling patients that change takes time, rather, patients need to know how to monitor change and how to get feedback from the scale, their bodies (e.g., fewer symptoms of strain, improved function, better fit of clothes), the environment, their physicians and peers. Additionally, the CSM suggests that this feedback will only result in continued engagement in healthy behaviors if it "makes sense." For example, while one outcome for any individual trying to lose weight will be the number on the scale, it may be equally, if not more important to create a record of intermediate outcomes that are clear benchmarks of progress, such as the presence of healthy foods in the house, individual healthy food choices, slowed eating, the enjoyment of healthy foods and the subjective sense of gains in ease of movement and vigor associated with even small improvements in physical function.

The CSM suggests that presenting weight loss as incremental will help patients focus on and be reinforced by the smaller steps that are necessary to both initiate and maintain weight loss. Each change would represent movement towards creating an environment in which healthy eating replaces the habit of unhealthy eating. Using an approach in which replacement makes use of the properties of old habits, but changes a few features, allows new and conscious decisions to co-opt and replace old, highly automatic habits that should maintain over time. For example, a patient could alter 
grocery-shopping habits to engender more healthful eating. That is, instead of automatically putting foods into the shopping cart, the patient would make food choice a conscious process. He or she would be instructed to look at nutrition labels (a habit which is associated with decreased calorie consumption [32]) and use that information to purchase foods, leaving healthy foods as "the available" choices when it's time to eat. The small steps involved in buying healthier foods on repeated shopping trips, will eventually result in a lower number on the scale. Observing a lower number on the scale in turn both validates the behavioral changes and "strengthens" the new habits (i.e., patients get proof that the new behaviors work). Thus, another potentially novel contribution of the CSM is the explicit incorporation of multiple feedback systems that integrate these habits into the sense of self. This provides coherence between specific actions and feelings of self-efficacy related to controlling diet, activity, and the ability to achieve a less heavy and healthier self in the present and future.

\section{Existing Research on the Common-Sense Model and Obesity}

Although many of the techniques described above are based on empirically tested relationships [33,34], none of the tests have been validated in the arena of weight regulation. A search of major scientific databases (e.g., PubMed, PsychInfo, MedLine, and ISI Web of Knowledge) for "common-sense model" and "obesity" resulted in zero citations. Knowing, however, that patients respond positively when physicians explore their "common-sense" perceptions about illnesses [27], signals a troublesome warning in relation to Ogden and Flanagan [23] findings that general practitioners (GPs) and lay people appear to have different beliefs about the causes of and treatments for obesity. Although both believed that behavioral factors caused obesity, significantly more lay people described biological factors as a significant causal factor for obesity while almost no GPs did. Further, while both groups were ambivalent about the effectiveness of obesity treatments, GPs were more likely to believe that behavioral treatments are indicated for obesity. Given GPs' beliefs that behavioral factors were critical causal factors for obesity, GPs' beliefs were more coherent. The authors suggest that GPs' stronger beliefs in behavioral treatments may actually inhibit them from counseling patients on weight loss: they may think it is not within their purview. Another study [35] found a high level of incoherence between beliefs about obesity and treatment among children-these children did not see inactivity as a cause for obesity, but did view increases in activity as treatment. It is possible that GPs may avoid confronting these discrepancies to avoid conflict and being seen as "blaming the patient."

To our knowledge, only two studies investigated relationships between obesity related illness beliefs and behaviors. The first [36], found that while perceiving obesity as chronic (time line), severe (consequences), and out of one's control was associated with less confidence in the ability to lose weight during a dietitian-led group weight loss program, none of these beliefs were associated with actual weight loss.
The second [37], which included over 3,500 participants, found that individuals who endorsed behavior as a cause of obesity reported higher levels of physical activity; whereas individuals who endorsed a genetic cause of obesity reported lower levels of physical activity and lower levels of fruit and vegetable consumption. Although there is clearly a need for more research to determine whether and how clinicians should use the CSM in practice, studies in areas other than weight control point to the potential value of assessing and augmenting representations of illness (e.g., obesity) and treatment (e.g., weight loss) during medical visits and in weight management programs as patients are more adherent to treatments when their illness and treatment beliefs are coherent [18] and addressed by clinicians $[28,38]$.

\section{How the Common-Sense Model Might Be Used in Clinical Practice}

We view CSM as a framework for integrating clinically validated procedures for behavioral change, rather than as a "stand alone" approach to weight loss. The following sections highlight some of the ways in which this integration can proceed in medical settings, though it could be applied to other venues, for example, specialty weight loss clinics.

Step one in applying the CSM involves the assessment of patients' representations of obesity (i.e., the five domains listed above and italicized below) and actions related to each of the five content areas, for example, causal actions, actions controlling onset or reducing obesity, time frames for outcomes, and actions controlling the diverse consequences of obesity. The information obtained from this assessment will provide insights into the patients' habits, for example, where they likely shop for food, what they eat, the foods they consider unhealthy and which they are willing and/or unwilling to change. The assessment may also provide clues regarding treatment preferences, untainted perhaps, by the "social desirability" present when patients respond to direct questions such as, "What would you do to reduce weight?" Patients' representations of obesity can also help clinicians understand patients' perceptions of the effectiveness of specific actions for controlling weight and their levels of selfefficacy for initiating and maintaining each. This information can be used to tailor treatments to fit patients' unique belief systems. Further, assessing the actions associated with the five domains can clarify motivations for change and suggest specific action plans for initiating and sustaining healthy behaviors [31]. We believe this process is best demonstrated by reviewing a sample visit between a female clinician and a male patient. Additionally, Table 1 provides suggestions for specific questions that can be used to assess each illness domain and help patients develop action plans. Necessary and corollary research is described in the next section.

\section{Describing Weight as a Health Threat}

It is critical to assess whether the patient identifies his weight as a threat to his health. A simple question, such as, "are you concerned about your weight?" is most likely sufficient to 
TABLE 1: Questions that can be used to assess patients' illness representations and action plans.

\begin{tabular}{|c|c|}
\hline Illness domain/action plan & Questions \\
\hline \multirow{3}{*}{ Identity } & Are you concerned about your weight? \\
\hline & Do you think you weigh too much? \\
\hline & Have others mentioned your weight? \\
\hline \multirow{2}{*}{ Cause } & What caused you to gain weight? \\
\hline & Why do you think you are overweight? \\
\hline \multirow{3}{*}{ Consequences } & How do you think your weight is affecting your health? \\
\hline & How do you think your weight will affect your health over the next few years? \\
\hline & $\begin{array}{l}\text { How do you think your weight is affecting your emotional wellbeing and your relationships with } \\
\text { others? }\end{array}$ \\
\hline \multirow{4}{*}{ Control/cure } & Have you tried anything to lose weight, or keep from gaining more weight? \\
\hline & How well has it worked? \\
\hline & What do you think you could do to control your weight? \\
\hline & What can you do to keep from gaining weight, or to lose weight? \\
\hline \multirow{3}{*}{ Time line } & How long have you felt that your weight has been a problem? \\
\hline & How long do you think it will take to change your eating/physical activity habits? \\
\hline & How long do you think it will take for you to lose weight? \\
\hline \multirow{4}{*}{ Action plan } & Can you pick one aspect of your diet or physical activity to work on before we next meet? \\
\hline & Where and when can that plan fit into your daily schedule? \\
\hline & What do you need in order to complete that plan? \\
\hline & How will you know whether the plan worked? \\
\hline
\end{tabular}

open the topic and begin assessment. Ideally, the patient will agree that his weight is a threat, however because his weight is a health threat-whether he recognizes it or not-it is important for the clinician to present it as such, for example, by describing the threat's identity (names, symptoms and functional declines) and time lines (e.g., at what age is loss of mobility most likely, diabetes and its consequences). She should also emphasize how these threats to quality of life and life itself can be reduced and hopefully avoided. By discussing these concerns, the clinician enriches the patient's illness representation of obesity by bringing his awareness to the experienced consequences of obesity, and likely time lines and causes. Further, the clinician can directly assess the patient's beliefs about these domains (consequences, control/cure, time lines, and causes) using concrete and direct questions, like those listed in Table 1. That is, she would use the CSM as a framework to guide the assessment inherent to any behavioral weight loss plan by focusing on what the CSM suggests are the most important beliefs when trying to affect weight.

\section{Creating an Action Plan}

A careful assessment of the patient's illness representation of obesity allows the clinician to create and share with the patient at least two distinct "maps": one of his life course with obesity and one of his daily patterns of eating, sitting, and moving about physically. The life-course map is an image for motivating and sustaining behavioral changes. The map of daily living depicts the setting in which to introduce these changes. The action plan that emerges from this latter step provides the clinician and the patient with information on the foods now consumed and identifies specific substitutes. It also provides a picture of the local environment in which to engage in new, specific physical activities. Thus, the patient's tailored action plan is built on his model of obesity and the features of his daily environment. For example, if the patient believes that inactivity is the cause of his current weight, he might be best served by focusing on ways to increase his activity levels, even at the expense of discussing food choices, as if he consumes the same number of calories, but expends more energy he should lose weight and perhaps more importantly, develop the sense of self-efficacy that can serve as a basis for changes in diet. Similarly, if the patient is completely unaware of how to control his weight, time may be best spent describing the relationship between calories consumed and energy expended or by referring the patient to a nutritional specialist.

It is important that the clinician use the development of the action plan as a time to integrate the patient's conscious choices and semiautomatic habits. It is also a time to clarify the problems and barriers in replacing existent, semiautomatic habits with conscious choices and the time frames for the latter to become semiautomatic. Thus, the effectiveness of an action plan, particularly its ability to sustain action over time, depends upon its fit between the patient's daily experiences and his conceptual or deliberative framework as well as the acceptance of occasional failures ( similar to strategies used in Relapse Prevention [39]). The clinician must ensure that the action plan instructs the patient on how to tell whether the treatment is working; for example, she can suggest ways for the patient to identify 
early evidence of the benefits of minor weight loss (e.g., rising from chairs more easily) and combine this with the self-monitoring that is often used in CBT treatments. In sum, a good action plan addresses several components of treatment representations as it identifies start points for action (control), validating cues (identity), and time lines for validation (consequences and control). This is especially important as eating is a repetitive action and control begins with the first bite of the day. The composition and quantity of that bite sets in motion processes assessing "progress," such as feeling full or wanting a snack. Increasing awareness of this pattern and the subjective cues driving the pattern will enhance control and improve the fit of the weight management action plan. The action plan is more likely to be internalized, that is, to be a cognitive, behavioral strategy for living, when it is personalized.

\section{Putting It All Together}

During a CSM-informed medical visit, the practitioner's task is to personalize assessment, counseling, and the construction of protocols for action for as many steps of the change process as possible. Thus, the clinician must describe the long-term goals of weight loss, that is, how much change is needed to avoid the most detrimental mental and physical outcomes of obesity. She must also identify pathways for moving towards a series of short and intermediate goals by clarifying start points; for example, by explaining that eating healthier begins by drawing up a shopping list before going to the super-market, not when one sits down to eat or that exercise begins by selecting a time and place to walk that fits into one's current sequence of activities. She should also describe time lines for monitoring progress. Further, the clinician must explain to the patient how and when to check progress (e.g., checking the amount of healthy food available in the home, monitoring weight once a week, and when and how to discuss lab results with a physician).

These techniques are similar to many of the behavioral techniques used in existing weight loss interventions (e.g., motivational interviewing [7] and behavioral weight loss treatments [6]). However, the primary strength of the CSM lies in the fact that it can help the clinician create an action plan framed by the patient's model of obesity and the behavioral treatments perceived to be most effective within that framework. This makes the risks of obesity concrete within each of the CSM content areas (e.g., the identities \{symptoms and functional damage\}, time of onset, and time living with proximal \{amputation and blindness for years and remote risks $\{$ death $\}$ ). This process also defines an action plan with pathways for specific behaviors that are nested in daily life activities. The entire process needs to be implemented as a personalized program for an active lifespan, which we hypothesize will improve initiation and adherence to weight loss protocols and the maintenance of these changes (i.e., sustained weight loss). We also hypothesize that it would be relatively easy to integrate these methods into existing treatments. Physicians often assess these domains during routine visits [28], so it should not be an excessive burden or time constraint. Indeed, using the
CSM as a framework for this assessment could streamline the process and save precious clinical time.

\section{Future Research}

The methods proposed above suggest several specific areas of future research on the CSM and obesity. First, we need more information on the basic components of commonsense models of obesity, that is, how people come, or fail to come, to feel and believe they are overweight, the risks they associate with overweight (what they are likely to experience, when and for how long), the procedures they perceive as effective for weight reduction and how they experience and judge the efficacy of their own and prescribed interventions. A better understanding of the evidence that patients use to judge efficacy (e.g., somatic experiences, time frames, consequences) and their interpretations of this evidence will allow investigators to relate those beliefs to motivation and action for initiating, sustaining or quitting specific weight reduction procedures and weight control programs.

This information should be acquired through a combination of qualitative studies embedded in a framework of hypothesis testing, longitudinal, and quantitative work that will lead to multicondition randomized experiments to test efficacy and effectiveness. For example, large crosssectional studies could correlate existing and empirically validated measures of illness beliefs (e.g., the various forms of the Illness Perception Questionnaire (e.g., [40, 41])) with outcomes such as weight, past attempts at weight loss, diet and physical activity. This information could be used to determine which illness and treatment beliefs and domains are most closely linked to necessary weight loss behaviors, which would suggest important areas for clinicians to target during assessment and treatment. This research could also include qualitative interviews that would begin to address the dynamic nature of the relationships among illness and treatment beliefs and health behaviors. Longitudinal studies will allow for more objective information regarding the dynamics among CSM-related beliefs and health behaviors. For example, tracking the beliefs, behaviors, and weights of individuals over time could explain whether certain beliefs are differentially associated with successful weight loss and maintenance.

We hypothesize that all five domains will be linked to health behaviors and outcomes and that the research described above will lead to the development of a weight loss specific CSM-based screener that clinicians could use to streamline assessment in clinical settings. As is the case with myocardial infarction [19], which has a similar treatment regimen to obesity, we hypothesize that the identity, control and consequence domains will be associated with initiating weight loss treatment and correlated with BMI. The time line and cause domains may be most important for maintaining weight loss as patients who consider weight loss behaviors as short term may be more likely to return to old, unhealthful habits once "treatment" ceases. Similarly, patients who do not consider lifestyle as a cause of obesity will most likely be nonadherent to lifestyle treatments as they will not make "common-sense." 
It will also be important to test for the efficacy and effectiveness of CSM-based techniques in clinical trials. As stated numerous times above, we hypothesize that the CSM will be most effective when combined with existing treatments. Therefore, the trials should compare an existing treatment with a CSM-enhanced version of that treatment (e.g., one that includes CSM informed assessment) to determine which is more effective at short and long-term weight loss. We hypothesize that there will be similar levels of effectiveness in short term weight loss, but that the CSMenhanced conditions will outperform standard treatment with regards to long term goals because the framework for change presented to patients will lead to changes that start as deliberate procedures, but that become automatic and effortless.

Similar research is already underway in at least two studies, one that uses the CSM to tailor weight loss interventions for patients on antipsychotic medications [42] and another that uses the CSM as the theoretical basis for an intervention to increase walking in patients with intermittent claudication [43]. The latter study is assessing illness beliefs and both studies could assess these beliefs at the beginning, middle and end of treatment and could then correlate CSM beliefs with outcomes and potentially test mediating effects. Having more information on the basics of patients' common-sense models could also provide clinicians with a variety of "shortcuts" during medical visits (e.g., if a specific illness domain is unrelated to outcomes, it need not be assessed).

\section{Conclusion}

The Common-Sense Model provides a framework for representing how people manage threats to health in everyday life; it is a "situated" cognitive behavioral model [44]. Therefore, in addition to providing a framework for conceptualizing and implementing clinical research across illnesses, the CSM has the additional virtue of addressing the language of the clinical encounter. It captures the intrapersonal processes involved in illness management as well as in the interpersonal discourse between patients and clinicians (in reviewing presenting problems and the formal review of systems, practitioners and patients use the language of the CSM). Thus, the CSM can track exchanges among patients and providers from diagnosis to the evaluation of treatment. This makes the CSM a self-regulation framework that is useful for increasingly precise predictions and is readily shared with clinicians and investigators interested in formalizing our approach to behavioral change for improving the health of individuals and populations. (We can be contacted for collaboration or further references.)

\section{Acknowledgments}

This paper was supported by the National Institute of Minority Health and Health Disparities (Grant R24 MD001691). The authors would like to thank the study team, members of the East Harlem Partnership for Diabetes Prevention Community Action Board, and the study participants.

\section{References}

[1] K. M. Flegal, M. D. Carroll, C. L. Ogden, and L. R. Curtin, "Prevalence and trends in obesity among US adults, 19992008," The Journal of the American Medical Association, vol. 303 , no. 3, pp. 235-241, 2010.

[2] National Institutes of Health, "Clinical Guidelines on the identification, evaluation, and treatment of overweight and obesity in adults- the evidence report," Obesity Research, vol. 6, supplement 2, pp. 51S-209S, 1998.

[3] World Health Organization, "Obesity and overweight," [Fact sheet no. 311], 2011, http://www.who.int/mediacentre/ factsheets/fs311/en/index.html.

[4] R. M. Puhl, "Bias, stigma and discrimination," in The Oxford Handbook of the Social Science of Obesity, J. Cawley, Ed., chapter 33, Oxford University Press, New York, NY, USA, 2011.

[5] E. A. Finkelstein, J. G. Trogdon, J. W. Cohen, and W. Dietz, "Annual medical spending attributable to obesity: payer-and service-specific estimates," Health Affairs, vol. 28, no. 5, pp. w822-w831, 2009.

[6] E. Loveman, G. K. Frampton, J. Shepherd et al., "The clinical effectiveness and costeffectiveness of long-term weight management schemes for adults: a systematic review," Health Technology Assessment, vol. 15, no. 2, pp. 1-182, 2011.

[7] M. J. Armstrong, T. A. Mottershead, P. E. Ronksley, R. J. Sigal, T. S. Campbell, and B. R. Hemmelgarn, "Motivational interviewing to improve weight loss in overweight and/or obese patients: a systematic review and meta-analysis of randomized controlled trials," Obesity Reviews, vol. 12, no. 1, pp. 709-723, 2011.

[8] T. Brown, A. Avenell, L. D. Edmunds et al., "Systematic review of long-term lifestyle interventions to prevent weight gain and morbidity in adults," Obesity Reviews, vol. 10, no. 6, pp. 627$638,2009$.

[9] A. R. Moldovan and D. David, "Effect of obesity treatments on eating behavior: psychosocial interventions versus surgical interventions. A systematic review," Eating Behaviors, vol. 12, no. 3, pp. 161-167, 2011.

[10] A. S. Poobalan, L. S. Aucott, E. Precious, I. K. Crombie, and W. C. S. Smith, "Weight loss interventions in young people (18 to 25 year olds): a systematic review," Obesity Reviews, vol. 11, no. 8, pp. 580-592, 2010.

[11] M. D. Witham and A. Avenell, "Interventions to achieve longterm weight loss in obese older people. A systematic review and meta-analysis," Age and Ageing, vol. 39, no. 2, Article ID afp251, pp. 176-184, 2010.

[12] W. C. Knowler, E. Barrett-Connor, S. E. Fowler et al., "Reduction in the incidence of type 2 diabetes with lifestyle intervention or metformin," The New England Journal of Medicine, vol. 346, no. 6, pp. 393-403, 2002.

[13] K. S. H. Yarnall, K. I. Pollak, T. Ostbye, K. M. Krause, and J. L. Michener, "Primary care: is there enough time for prevention?" American Journal of Public Health, vol. 93, no. 4, pp. 635-641, 2003.

[14] A. G. Tsai and T. A. Wadden, "Treatment of obesity in primary care practice in the United States: a systematic review," Journal of General Internal Medicine, vol. 24, no. 9, pp. 1073-1079, 2009.

[15] H. Leventhal, S. Bodnar-Deren, J. Y. Breland et al., "Modeling health and illness behavior: the approach of the commonsense model," in Handbook of Health Psychology, A. Baum, T. A. Revenson, and J. Singer, Eds., pp. 3-36, Psychological Press, New York, NY, USA, 2nd edition, 2012. 
[16] C. Clar, K. Barnard, E. Cummins, P. Royle, and N. Waugh, "Self-monitoring of blood glucose in type 2 diabetes: systematic review," Health Technology Assessment, vol. 14, no. 12, pp. $1-140,2010$.

[17] U. L. Malanda, L. M. C. Welschen, I. I. Riphagen, J. M. Dekker, G. Nijpels, and S. D. M. Bot, "Self-monitoring of blood glucose in patients with type 2 diabetes mellitus who are not using insulin (Review)," Cochrane Database of Systematic Reviews, no. 1, Article ID CD005060, 2012.

[18] M. S. Hagger and S. Orbell, "A meta-analytic review of the common-sense model of illness representations," Psychology and Health, vol. 18, no. 2, pp. 141-184, 2003.

[19] D. P. French, A. Cooper, and J. Weinman, "Illness perceptions predict attendance at cardiac rehabilitation following acute myocardial infarction: a systematic review with metaanalysis," Journal of Psychosomatic Research, vol. 61, no. 6, pp. 757-767, 2006.

[20] K. J. Petrie, L. D. Cameron, C. J. Ellis, D. Buick, and J. Weinman, "Changing illness perceptions after myocardial infarction: an early intervention randomized controlled trial," Psychosomatic Medicine, vol. 64, no. 4, pp. 580-586, 2002.

[21] E. A. Halm, P. Mora, and H. Leventhal, "No symptoms, no asthma: the acute episodic belief is associated with poor selfmanagement among inner-city adults with persistent asthma," Chest, vol. 129, no. 3, pp. 573-580, 2006.

[22] A. A. Kaptein, B. M. Hughes, M. Scharloo et al., "Illness perceptions about asthma are determinants of outcome," Journal of Asthma, vol. 45, no. 6, pp. 459-464, 2008.

[23] J. Ogden and Z. Flanagan, "Beliefs about the causes and solutions to obesity: a comparison of GPs and lay people," Patient Education and Counseling, vol. 71, no. 1, pp. 72-78, 2008.

[24] M. R. Goldfried, Clinical Behavior Therapy, John Wiley \& Sons, New York, NY, USA, 1976.

[25] G. T. Wilson, "Rapid response to cognitive behavior therapy," Clinical Psychology: Science and Practice, vol. 6, no. 3, pp. 289292, 1999.

[26] K. MacGregor, M. Handley, S. Wong et al., "Behavior-change action plans in primary care: a feasibility study of clinicians," Journal of the American Board of Family Medicine, vol. 19, no. 3, pp. 215-223, 2006.

[27] D. T. D. de Ridder, N. C. M. Theunissen, and S. M. van Dulmen, "Does training general practitioners to elicit patients' illness representations and action plans influence their communication as a whole?" Patient Education and Counseling, vol. 66, no. 3, pp. 327-336, 2007.

[28] L. A. Phillips, H. Leventhal, and E. A. Leventhal, "Physicians' communication of the common-sense self-regulation model results in greater reported adherence than physicians' use of interpersonal skills," British Journal of Health Psychology, vol. 17, no. 2, pp. 244-257, 2012.

[29] F. Hill-Briggs, M. Lazo, M. Peyrot et al., "Effect of problemsolving-based diabetes self-management training on diabetes control in a low income patient sample," Journal of General Internal Medicine, vol. 26, no. 9, pp. 972-978, 2011.

[30] H. Leventhal, R. Singer, and S. Jones, "Effects of fear and specificity of recommendation upon attitudes and behavior," Journal of Personality and Social Psychology, vol. 2, no. 1, pp. 20-29, 1965.

[31] K. Witte and M. Allen, "A meta-analysis of fear appeals: implications for effective public health campaigns," Health Education and Behavior, vol. 27, no. 5, pp. 591-615, 2000.
[32] C. A. Roberto, M. B. Schwartz, and K. D. Brownell, "Rationale and evidence for menu-labeling legislation," American Journal of Preventive Medicine, vol. 37, no. 6, pp. 546-551, 2009.

[33] H. Leventhal, E. A. Leventhal, and J. Y. Breland, "Cognitive science speaks to the "Common-Sense" of chronic illness management," Annals of Behavioral Medicine, vol. 41, no. 2, pp. 152-163, 2011.

[34] P. M. Gollwitzer, "Implementation intentions: strong effects of simple plans," American Psychologist, vol. 54, no. 7, pp. 493503, 1999.

[35] M. Babooram, B. A. Mullan, and L. Sharpe, "Children's perceptions of obesity as explained by the common sense model of illness representation," British Food Journal, vol. 113, no. 2, pp. 234-247, 2011.

[36] Y. Benyamini and O. Raz, "'I can tell you if i'll really lose all that weight': dispositional and situated optimism as predictors of weight loss following a group intervention," Journal of Applied Social Psychology, vol. 37, no. 4, pp. 844-861, 2007.

[37] C. Wang and E. J. Coups, "Causal beliefs about obesity and associated health behaviors: results from a populationbased survey," International Journal of Behavioral Nutrition and Physical Activity, vol. 7, article 19, 2010.

[38] L. A. Phillips, E. A. Leventhal, and H. Leventhal, "Factors associated with the accuracy of physicians' predictions of patient adherence," Patient Education and Counseling, vol. 85, no. 3, pp. 461-467, 2011.

[39] J. R. Gordon and G. A. Marlatt, Eds., Relapse Prevention: Maintenance Strategies in the Treatment of Addictive Behaviors, Guilford Press, New York, NY, USA, 1985.

[40] J. Weinman, K. J. Petrie, R. Moss-Morris, and R. Horne, "The illness perception questionnaire: a new method for assessing the cognitive representation of illness," Psychology and Health, vol. 11, no. 3, pp. 431-445, 1996.

[41] E. Broadbent, K. J. Petrie, J. Main, and J. Weinman, "The brief illness perception questionnaire," Journal of Psychosomatic Research, vol. 60, no. 6, pp. 631-637, 2006.

[42] T. Bradshaw, A. Wearden, M. Marshall et al., "Developing a healthy living intervention for people with early psychosis using the Medical Research Council's guidelines on complex interventions: phase 1 of the HELPER-interACT programme," International Journal of Nursing Studies, vol. 49, no. 4, pp. 398-406, 2012.

[43] M. A. Cunningham, V. Swanson, R. E. O'Carroll, and R. J. Holdsworth, "Increasing walking in patients with intermittent claudication: protocol for a randomised controlled trial," BMC Cardiovascular Disorders, vol. 10, article 49, 2010.

[44] J. G. Greeno, "The situativity of knowing, learning, and research,” American Psychologist, vol. 53, no. 1, pp. 5-26, 1998. 


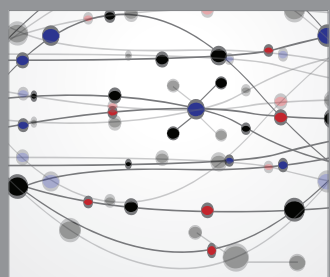

The Scientific World Journal
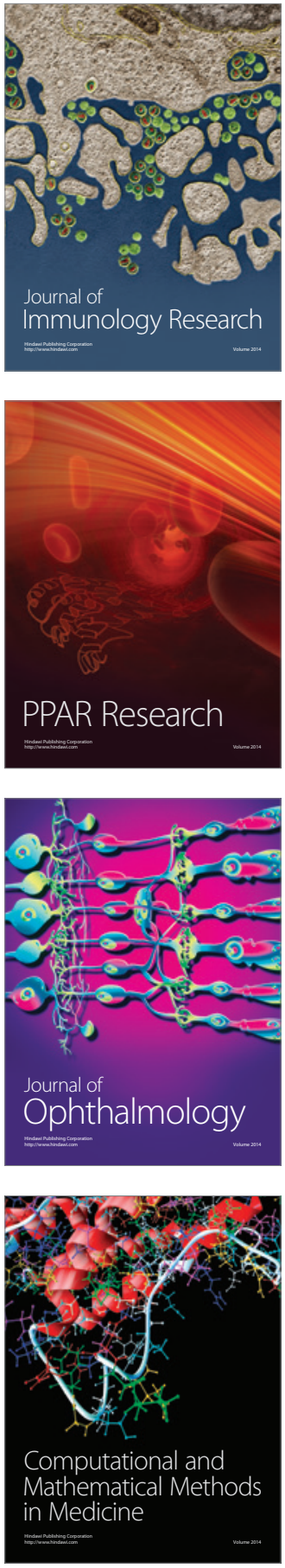

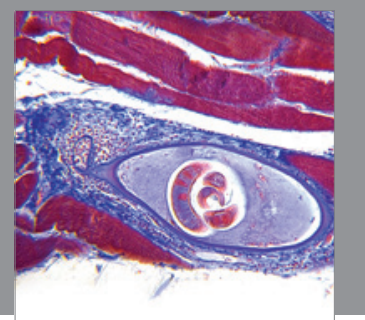

Gastroenterology

Research and Practice
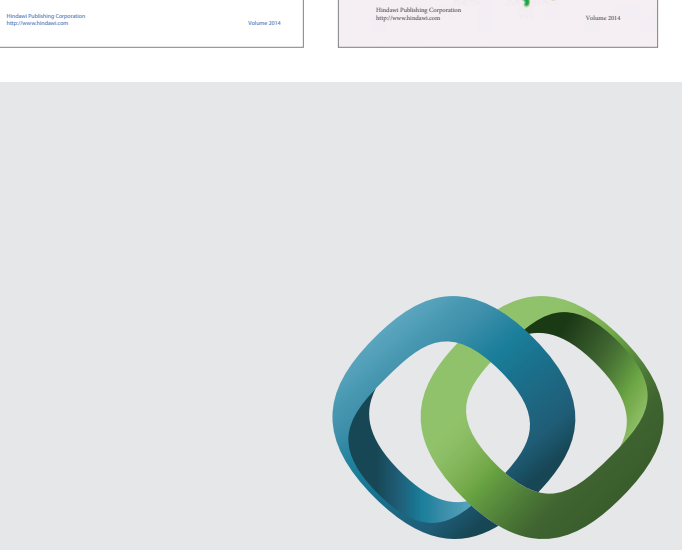

\section{Hindawi}

Submit your manuscripts at

http://www.hindawi.com
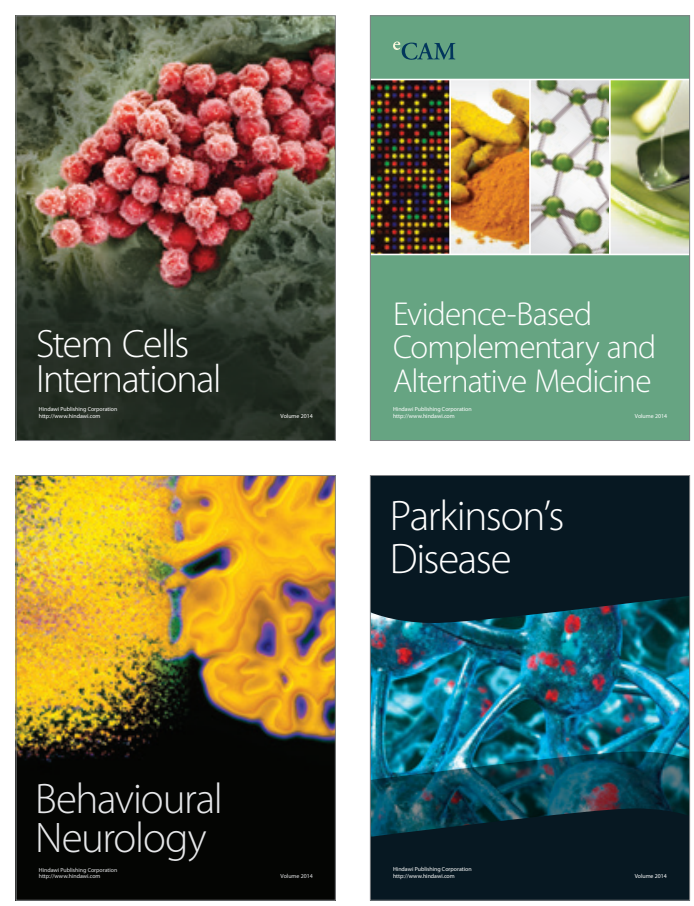

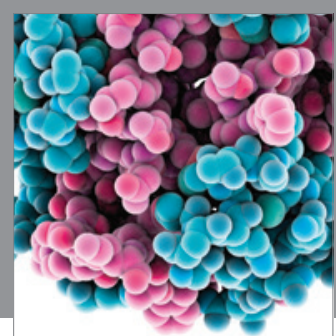

Journal of
Diabetes Research

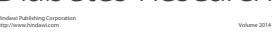

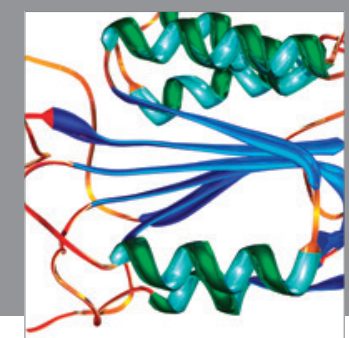

Disease Markers
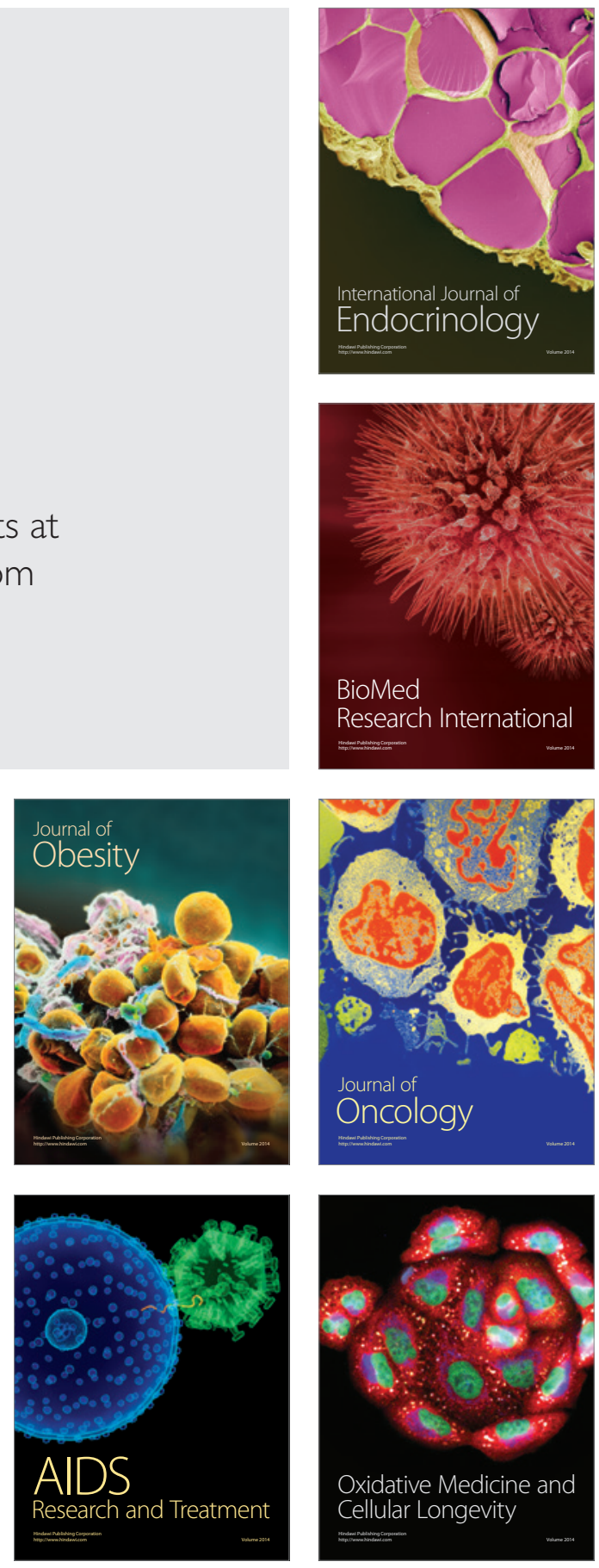